\title{
Postoperative Pancreatic Fistula after a Left Cytoreductive Nephrectomy
}

\author{
Bharath N Kumar ${ }^{1}$, Sanjeev Tandon ${ }^{2}$, Irshad A Khan ${ }^{3}$, Aditya A Jha ${ }^{4}$
}

\begin{abstract}
Aim: The aim of the study was to report a case of postoperative pancreatic fistula (POPF) in the setting of a left cytoreductive nephrectomy. Background: Pancreatic injury during nephrectomy is a rare complication with a reported incidence of $2.1 \%$ in laparoscopic left radical nephrectomy. It has not been described in the setting of cytoreductive nephrectomy (CN) and we report one such case.

Case description: A 61-years-old male underwent left $\mathrm{CN}$ for metastatic left renal mass. On the 5th postoperative day, there was spontaneous expulsion of the retroperitoneal drain, two days after which the patient developed a serous discharge of $25-30 \mathrm{~mL}$ per day from the drain site, which later became purulent. The patient was managed successfully with pigtail drainage of the collection, culture-sensitive antibiotics, and subcutaneous injection of octreotide.

Conclusion: Conservative management of POPF after nephrectomy in the form of drainage of retroperitoneal collection, antibiotics, somatostatin analogs, and discontinuing oral diet with total parenteral nutrition (TPN) (as indicated) is successful in most cases.

Clinical significance: A pancreatic injury during a left $\mathrm{CN}$ is a distinct possibility, which can be managed successfully by conservative means. Keywords: Case report, Left cytoreductive nephrectomy, Metastatic renal cell carcinoma, Postoperative pancreatic fistula. Journal of Medical Academics (2019): 10.5005/jp-journals-10070-0026
\end{abstract}

\section{BACKGROUND}

A pancreatic injury during a nephrectomy is a rare complication with a reported incidence of $2.1 \%$ in laparoscopic left radical nephrectomies. ${ }^{1}$ It has not been described in the setting of a $\mathrm{CN}$ and we present one such case.

\section{Case Description}

A 61-years-old male presented with gross, total, painless hematuria. Imaging revealed a large upper polar left renal mass with illdefined fat planes with tail of pancreas, distal spleen, and left adrenal gland, along with multiple bilateral lung nodules and mediastinal lymph nodes (Fig. 1). The patient underwent a left CN through the flank approach and intraoperatively found to have a large upper polar left renal mass, closely abutting the tail of pancreas. On the 5th postoperative day, there was a spontaneous expulsion of the retroperitoneal drain, two days after which the patient developed a serous discharge of about $25-30 \mathrm{~mL}$ per day from the drain site, which later became purulent associated with high-grade fever. Imaging with contrast-enhanced computerized tomography revealed bulky tail of pancreas and fluid collection in the surgical bed tracking down to drain site (Fig. 2). Drain fluid as well as serum amylase and lipase were raised (Table 1). Drain fluid culture grew Pseudomonas. Patient was managed with pigtail drainage of the collection, culture-sensitive antibiotics, and subcutaneous injection of octreotide. The fever resolved 10 days after pigtail drainage. The drain fluid amount decreased gradually, but was still persisting at 5-10 mL per day. Patient was discharged on the 43rd postoperative day and the drain was removed on the 70th postoperative day after gradual withdrawal, with no adverse events. A positron emission tomography scan revealed persistence of lung and mediastinal lymph node metastases and patient was started on Sunitinib.
${ }^{1-4}$ Department of Urology, Base Hospital, New Delhi, India

Corresponding Author: Bharath N Kumar, Department of Urology, Base Hospital, New Delhi, India, Phone: +91 9871927128, e-mail: drbharathkumarn@gmail.com

How to cite this article: Kumar BN, Tandon S, et al. Postoperative Pancreatic Fistula after a Left Cytoreductive Nephrectomy. J Med Acad 2019;2(1):33-35.

Source of support: Nil

Conflict of interest: None

\section{Discussion}

About $30 \%$ of renal cell carcinoma (RCC) patients present with metastases at diagnosis and the 5 -year survival rate ranges from $0 \%$ to $20 \%$ for such patients. Debulking surgery in the form of $\mathrm{CN}$ in selected patients of metastatic RCC (mRCC) followed by treatment with tyrosine kinase inhibitors of vascular endothelial growth factor (VEGF) receptor remains the treatment of choice. Complications of $\mathrm{CN}$ include injury to adjacent structures such as bowel, liver, spleen, and pancreas depending on the side of nephrectomy.

Pancreatic injury is a rare complication of nephrectomy, particularly an injury to the tail of the pancreas in a left nephrectomy because of the proximity of this organ, especially with difficult dissection or in the presence of a large tumor. The reason for POPF formation is intraoperative damage to pancreatic tissue and opening of pancreatic ducts. The key to avoiding pancreatic injuries is to completely mobilize the spleen and pancreas en bloc. In our case, the large upper polar left renal mass was very close to the tail of the pancreas, which may have been the reason for this complication. If the injury is intraoperatively identified and the damage minimal and pancreatic duct is intact, then a conservative approach is followed with placement of an intraperitoneal drain. If the lesion is 


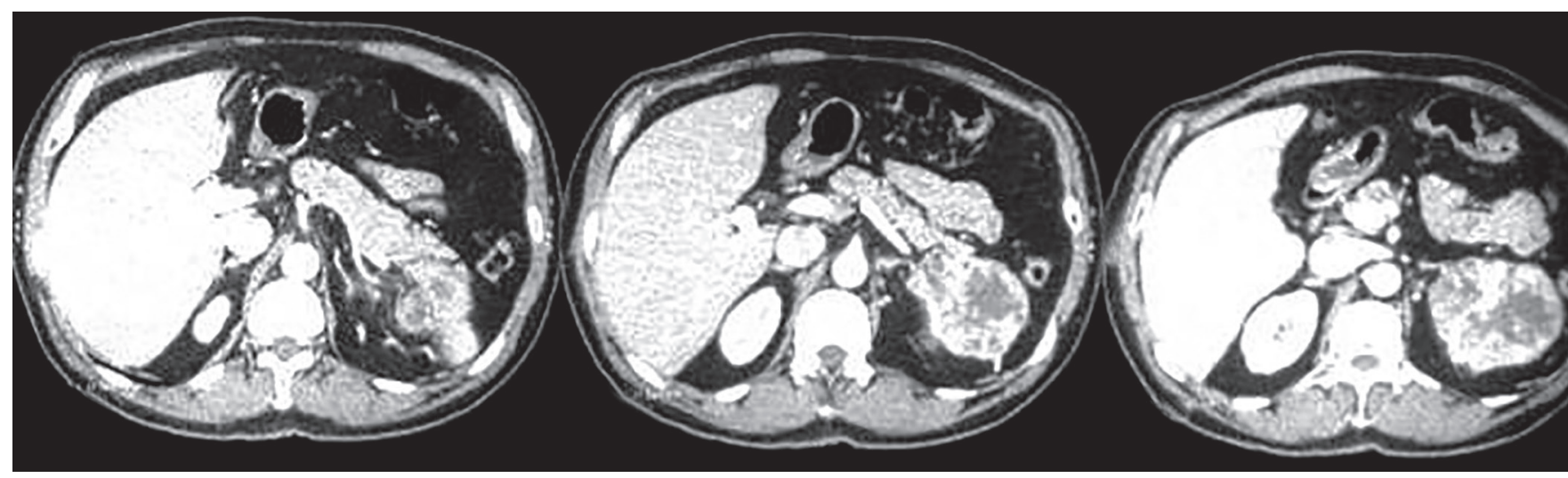

Fig. 1: Preoperative CECT Abdomen showing left renal tumour closely abutting tail of pancreas

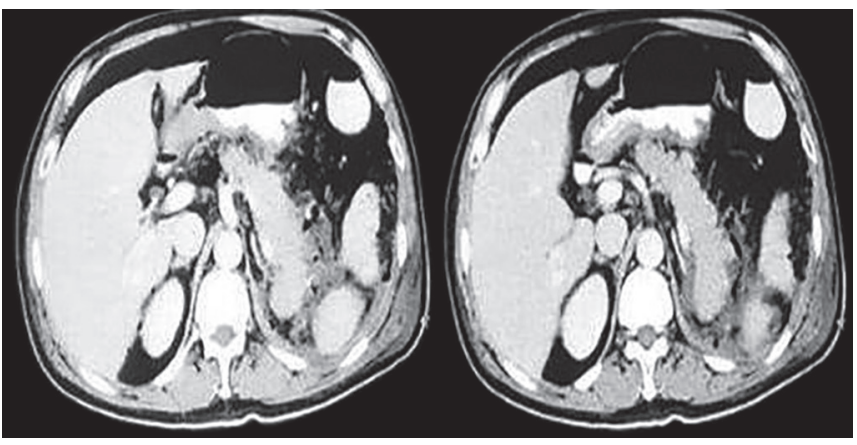

Fig. 2: Postoperative CECT abdomen showing bulky tail of pancreas with fluid collection

Table 1: Trend of serum and drain fluid enzymes

\begin{tabular}{llllll}
\hline & \multicolumn{2}{c}{ Drain } & & \multicolumn{2}{c}{ Serum } \\
\cline { 2 - 3 } \cline { 5 - 6 } $\begin{array}{l}\text { Postoperative } \\
\text { day (POD) }\end{array}$ & $\begin{array}{l}\text { Amylase } \\
(I U / L)\end{array}$ & Lipase (IU/L) & & $\begin{array}{l}\text { Amylase } \\
(I U / L)\end{array}$ & Lipase (IU/L) \\
\hline 12th POD & 315 & 3,500 & & 378 & 3,755 \\
15th POD & 350 & 2,709 & & 304 & 2,548 \\
17th POD & 318 & 2,660 & & 237 & 1,696 \\
\hline
\end{tabular}

deep and crosses the midline of the tail of the pancreas, suggesting a lesion of the main pancreatic duct, then distal pancreatectomy is the safe option. ${ }^{2}$ However, unfortunately, the majority of the cases are recognized postoperatively. The patient usually presents with abdominal pain, elevated serum amylase and lipase, and fluid collection on a computed tomography (CT) scan. In our case, despite placement of a drain, unfortunately it got spontaneously expelled, leading to the POPF.

Postoperative pancreatic fistula is classified into three grades as per the 2016 update of the International Study Group on Pancreatic Surgery (ISGPS) definition and grading of POPF. The first grade is known as "Biochemical Leak" in which the drain fluid amylase is more than three times the upper limit of institutional normal serum amylase; there is no deviation in the normal postoperative pathway and normal postoperative duration of stay; the drain may remain in place even after discharge for observation purposes for up to 3 weeks after operation. In grade II POPF, there would be a change in management of expected postoperative pathway, persistent drainage even 3 weeks after operation, requirement of percutaneous or endoscopic drainage, occurrence of POPF-related hemorrhage, or pseudo-aneurysm requiring transfusions and/or angiography; there may be signs of infection without organ failure. Grade III POPF is when patient requires reoperation, develops organ failure, or ends up in death. ${ }^{3}$ Our patient had developed grade II POPF.

Conservative management in the form of drainage of retroperitoneal collection, culture-sensitive antibiotics, somatostatin analogs, discontinuing oral diet, and placing the patient on TPN is successful in $30-50 \%$ of cases. ${ }^{4}$ We did not discontinue oral diet or start TPN in our patient, since the fistula output was very low. Spontaneous closure of the fistula with this conservative approach is usually achieved within $33-55$ days. ${ }^{4}$ We removed the drain only on the 70th postoperative day to be on the safer side, though the daily output was only about $5-10 \mathrm{~mL}$ for many days. In retrospect we believe that the drain could have been removed earlier without any adverse effect on outcome.

If conservative management fails, then more invasive treatment in the form of endoscopic sphincterectomy of or prosthetic restoration of Wirsung's duct may be required. Free outflow of the pancreatic fluid to the duodenum instead of the canal of the fistula is enabled through the prosthesis. The time of closure with this method of treatment is usually 15-39 days. If after 6-8 weeks the fistula remains active, surgery is indicated in the form of a drainage surgery such as fistulojejunostomy or resection of tail of pancreas.

Bozkurt et al. reported a patient of POPF presenting 70 days after a laparoscopic left radical nephrectomy, who responded successfully to conservative management. ${ }^{5}$

\section{Conclusion}

Conservative management of POPF after nephrectomy in the form of drainage of retroperitoneal collection, antibiotics, somatostatin analogs, and discontinuing oral diet with TPN (as indicated) is successful in $30-50 \%$ of cases. Intervention in the form of endoscopic sphincterectomy, prosthetic restoration of Wirsung's duct, fistulojejunostomy, or resection of tail of pancreas is required in recalcitrant cases. 


\section{Clinical Significance}

Pancreatic injury during left $\mathrm{CN}$ is a distinct possibility, which can be managed successfully by conservative means.

\section{References}

1. Varkarakis IM, Allaf ME, et al. Pancreatic injuries during laparoscopic urologic surgery. Urology 2004;64:1089-1093. DOI: 10.1016/ j.urology.2004.06.032.

2. Breda A, Finelli A, et al. Complications of laparoscopic surgery for renal masses: prevention, management, and comparison with the open experience. Eur Urol 2009;55(4):836-850. DOI: 10.1016/ j.eururo.2009.01.018.

3. Bassi C, Marchegiani G, et al. The 2016 update of the International Study Group (ISGPS) definition and grading of postoperative pancreatic fistula: 11 Years After. Surgery 2017;161(3):584-591. DOI: 10.1016/j.surg.2016.11.014.

4. Bassi C, Dervenis C, et al. Postoperative pancreatic fistula: an international study group (ISGPF) definition. Surgery 2005;138(1): 8-13. DOI: 10.1016/j.surg.2005.05.001.

5. Bozkurt M, Can O, et al. A Pancreatic Fistula as a Rare Complication of Laparascopic Radical Nephrectomy: A Case Report. Urol Case Rep 2017;12:20-22. DOI: 10.1016/j.eucr.2017.02.004. 